\title{
A Derivation Of The Scalar Propagator In A Planar Model In Curved Space
}

\author{
S. G. Kamath* \\ Department of Mathematics, Indian Institute of Technology Madras, Chennai 600 036, India \\ *e-mail: kamath@iitm.ac.in
}

Abstract: Given that the free massive scalar propagator in $2+1$ dimensional Euclidean space is $D(x-y)=\frac{1}{4 \pi \rho} e^{-m \rho}$ with $\rho^{2}=(x-y)^{2}$ we present the counterpart of $D(x-y)$ in curved space with a suitably modified version of the Antonsen - Bormann method instead of the familiar Schwinger - de Witt proper time approach, the metric being defined by the rotating solution of Deser et al. of the Einstein field equations associated with a single massless spinning particle located at the origin.

Keywords: massive scalar field theory, $2+1$ dimensions, curved space - time, stationary solutions, heat kernel, propagator.

PACS : $04.60 \mathrm{Kz}, 04.62+\mathrm{v}, 11.10 \mathrm{Kk}$

\section{INTRODUCTION}

This is the second of two papers associated with the Lagrangian density

$$
L=\frac{1}{2} g^{\mu \rho} \partial_{\mu} \varphi \partial_{\rho} \varphi-\frac{1}{2} m^{2} \varphi^{2}
$$

in $2+1$ dimensions; it extends the scope of the first [1] with this objective: Derive in curved space the counterpart of the propagator $\langle 0| T^{*}(\varphi(x) \varphi(y)|0\rangle$ that is given in Euclidean flat space - with $g^{\alpha \beta}=\operatorname{diag}(1,1,1)-$ by $D(x-y)=\frac{1}{4 \pi \rho} e^{-m \rho}$ with $\rho^{2}=(x-y)^{2}$. This report is inspired by two recent papers of the Napoli group of Bimonte et al.[2,3] and is based on the method proposed by Antonsen and Bormann[4,5]. To elaborate, we recall here that Bimonte et al.[2] obtain the Green's functions in curved space using the Fock - Schwinger - de Witt method[6,7,8], an important feature of which is that it is valid only in the asymptotic region - see eq.(2.4) in Ref.2 et seq.. Again, the use of the Fermi normal coordinates in Bunch and Parker[9] leads to a momentum space representation for the Feynman propagator in curved space but the said infirmity that obtains in [2] still prevails, while the recent calculation[3] of the energy momentum tensor for a Casimir apparatus but in a weak gravitational field using a Fermi coordinate system only reinforces our aim. In this connection, the work of Antonsen and 
Bormann $[4,5]$ holds some promise but with a modification that is imperative for our purposes, given that in Ref.4 the last term in eq.(23) has been set to zero[10] thus undermining the utility of their method[4,5].

\section{The Antonsen - Bormann Method}

We begin with the operator $B \equiv-\partial^{\mu}\left(g_{\mu \rho} \partial^{\rho}\right)-m^{2}$ obtained from eq.(1) above and work with the heat kernel $G\left(x, x^{\prime} ; \sigma\right)$ obeying

$$
B G\left(x, x^{\prime} ; \sigma\right)=-\frac{\partial G}{\partial \sigma}
$$

with $G\left(x, x^{\prime} ; \sigma \rightarrow 0\right)=\delta^{(3)}\left(x-x^{\prime}\right)$. The vierbeins $e_{\beta}^{\alpha}$ defined by $g^{\alpha \beta}=\eta^{\mu v} e_{\mu}^{\alpha} e_{v}^{\beta}$ with $\eta^{\mu v}=$ $\operatorname{diag}(1,-1,-1)$ can now be used to rework $B$ into the form

$$
B=-\eta^{a b} \partial_{a} \partial_{b}-m^{2}-e_{\alpha}^{m} \partial_{m}\left(e_{a}^{\alpha}\right) \partial^{a}
$$

Writing $G\left(x, x^{\prime} ; \sigma\right)$ in terms of the flat space heat kernel $G_{0}\left(x, x^{\prime} ; \sigma\right)$ as

$$
G\left(x, x^{\prime} ; \sigma\right)=G_{0} e^{-\frac{1}{2} \int e_{\alpha}^{m} \partial_{m}\left(e_{n}^{\alpha}\right) d x^{n}} e^{-T}
$$

with $\left(-\eta^{a b} \partial_{a} \partial_{b}-m^{2}\right) G_{0}=-\frac{\partial G_{0}}{\partial \sigma}$ and $G_{0}\left(x, x^{\prime} ; \sigma \rightarrow 0\right)=\delta^{(3)}\left(x-x^{\prime}\right)$ enables us to remove the last term in (3) and obtain as in Ref.4

$$
\partial^{a} \partial_{a} T-\partial^{a} T \partial_{a} T+f+2 \frac{\partial^{\mu} G_{0}}{G_{0}} \partial_{\mu} T=\frac{\partial T}{\partial \sigma}
$$

with $f=\frac{1}{4}\left(e_{\alpha}^{m} \partial_{(}\left(e_{b}^{\alpha}\right)\right)^{2}+\frac{1}{2} \partial^{n}\left(e_{\beta}^{m} \partial_{m}\left(e_{n}^{\beta}\right)\right)$. The last term in (5) can be reworked as $-\frac{\left(x-x^{\prime}\right)^{a}}{\sigma} \partial_{a} T$ recalling that in Euclidean space $G_{0}=(4 \pi \sigma)^{-3 / 2} e^{-\frac{\left(x-x^{\prime}\right)^{2}}{4 \sigma}-m^{2} \sigma}$. We shall now write $\mathrm{T}$ as

$$
T=\frac{\tau_{-1}}{\sigma}+\sum_{k=0}^{\infty} \tau_{k} \sigma^{k}
$$

and not as in eq.(24) in Ref.4 to meet the twin requirements of : a. the boundary condition on the heat kernels $\mathrm{G}$ and $G_{0}$ as $\sigma \rightarrow 0$ thus setting $\tau_{0}=-\frac{1}{2} \int e_{\alpha}^{n} \partial_{n}\left(e_{m}^{\alpha}\right) d x^{m}$ and b. retaining the extra term $-\frac{\left(x-x^{\prime}\right)^{a}}{\sigma} \partial_{a} T$. Repeating the steps in Ref.4 one now gets $\tau_{-1}$ to be a solution of

$$
\partial^{a} \partial_{a} \tau_{-1}-2 \partial^{a} \tau_{0} \partial_{a} \tau_{-1}-\left(x-x^{\prime}\right)^{a} \partial_{a} \tau_{0}=0
$$

Besides eq.(6) here are a few more entries that are the counterparts of those given by eq.(14) in Ref.5:

$$
\begin{gathered}
2 \partial^{a} \tau_{-1} \partial_{a} \tau_{1}+\left(x-x^{\prime}\right)^{a} \partial_{a} \tau_{1}-f+\tau_{1}+\partial^{a} \tau_{0} \partial_{a} \tau_{0}-\partial^{a} \partial_{a} \tau_{0}=0 \\
\partial^{a} \partial_{a} \tau_{1}-2 \partial_{a} \tau_{-1} \partial^{a} \tau_{2}-2 \partial_{a} \tau_{1} \partial^{a} \tau_{0}-\left(x-x^{\prime}\right)^{a} \partial_{a} \tau_{2}=2 \tau_{2}
\end{gathered}
$$




\section{Some Examples of the Vierbeins $e_{a}^{\mu}$ and $e_{\mu}^{a}$}

Adopting the stationary solutions of the Einstein field equations given in Deser, Jackiw and 'tHooft[11] and Clement[12] wherein

$$
g^{00}=1-\frac{\lambda^{2}}{r^{2}}, g^{01}=-\frac{\lambda y}{r^{2}}, g^{02}=\frac{\lambda x}{r^{2}}, g^{11}=-1, g^{12}=0, g^{22}=-1
$$

with $r=|\boldsymbol{r}|$, it is easy to determine the vierbeins $e_{a}^{\mu}$; their counterparts $e_{\mu}^{a}$ are got from $g_{\alpha \beta}=\eta_{\mu \nu} e_{\alpha}^{\mu} e_{\beta}^{\nu}$ where $g_{\alpha \beta}$ is given by[11,12]

$$
g_{00}=1, g_{01}=-\frac{\lambda y}{r^{2}}, g_{02}=\frac{\lambda x}{r^{2}}, g_{11}=-1+\left(\frac{\lambda y}{r^{2}}\right)^{2}, g_{12}=-\left(\frac{\lambda^{2} x y}{r^{4}}\right), g_{22}=-1+\left(\frac{\lambda x}{r^{2}}\right)^{2}
$$

with $2 \pi \lambda=\kappa \mathrm{J}, \kappa=8 \pi G$ being the gravitational constant and $\mathrm{J}=|\mathrm{J}|$ being the spin of the massless particle located at the origin(see eq.(20) in Clement[12]); note that $\operatorname{det}_{\mu \nu}=1$ so the action $S=\int d^{3} x L$ with $L$ as in (1). As an example for which $\tau_{0}=0$ and $f=0$ we have:

$$
\begin{aligned}
& e_{a}^{\mu}: e_{0}^{0}=\frac{i \lambda y}{r^{2}}, e_{0}^{1}=i, e_{0}^{2}=0 ; e_{1}^{0}=i, e_{1}^{1}=0, e_{1}^{2}=0 ; e_{2}^{0}=\frac{\lambda x}{r^{2}}, e_{2}^{1}=0, e_{2}^{2}=-1 \\
& e_{\mu}^{a}: e_{0}^{0}=1, e_{0}^{1}=0=e_{0}^{2} ; e_{1}^{0}=-\frac{\lambda y}{r^{2}}, e_{1}^{1}=\frac{1}{\sqrt{2}}, e_{1}^{2}=-\frac{1}{\sqrt{2}} ; e_{2}^{0}=\frac{\lambda x}{r^{2}}, e_{2}^{1}=-\frac{1}{\sqrt{2}}, e_{2}^{2}=-\frac{1}{\sqrt{2}}
\end{aligned}
$$

An example which yields $\tau_{0}=\frac{i \lambda x}{2 r^{2}}$ and $f=\frac{\lambda^{2}}{4 r^{4}}$ is :

$$
\begin{aligned}
& e_{a}^{\mu}: e_{0}^{0}=1, e_{0}^{1}=0, e_{0}^{2}=0 ; e_{1}^{0}=\frac{\lambda x}{r^{2}}, e_{1}^{1}=0, e_{1}^{2}=-1 ; e_{2}^{0}=\frac{\lambda y}{r^{2}}, e_{2}^{1}=1, e_{2}^{2}=0 \\
& e_{\mu}^{a}: e_{0}^{0}=0, e_{0}^{1}=-i, e_{0}^{2}=0 ; e_{1}^{0}=\frac{i}{\sqrt{2}}, e_{1}^{1}=\frac{i \lambda y}{r^{2}}, e_{1}^{2}=\frac{1}{\sqrt{2}} ; e_{2}^{0}=\frac{i}{\sqrt{2}}, e_{2}^{1}=-\frac{i \lambda x}{r^{2}}, e_{2}^{2}=-\frac{1}{\sqrt{2}}
\end{aligned}
$$

It is easy to check for this example that $\partial^{a} \partial_{a} \tau_{0}=0$ and that $\partial_{a} \tau_{0} \partial^{a} \tau_{0}=\frac{\lambda^{2}}{4 r^{4}}$, which is $f$ above. Clearly, it is not difficult to cite other examples involving for instance the circular functions[13].

\section{Solving Eqs.(6) - (8) Above}

With $\tau_{-1}=\chi_{-1} e^{\tau_{0}}$ one gets from (6)

$$
\partial^{a} \partial_{a} \chi_{-1}+\left(\partial^{a} \partial_{a} \tau_{0}-2 \partial_{a} \tau_{0} \partial^{a} \tau_{0}\right) \chi_{-1}=\left(x-x^{\prime}\right)^{a} \partial_{a} \tau_{0} e^{-\tau_{0}}
$$

the solution to which can be attempted using a Green's function, thus yielding $\tau_{-1}$. The learned reader will notice that with the coefficient of $\chi_{-1}$ above set at $\frac{\lambda^{2}}{2 r^{4}},(11)$ now becomes an inhomogeneous Helmholtz equation[14] in Minkowski space. With $f$ and $\tau_{-1}$ known the method of Lagrange readily yields a solution to (7), it being a first order partial differential equation; thus $\tau_{1}$ is obtained. 
$\tau_{2}$ can now be got from(8) since the latter is also a first order pde. This exercise can now be repeated to obtain the $\tau_{j}, j \geq 3$ resulting in the full form of T above and thus the heat kernel $G\left(x, x^{\prime} ; \sigma\right)$, whose integral - see eq.(5) in Ref. 5 - yields the required propagator that is the counterpart of $D(x-y)$ in curved space.

\section{ACKNOWLEDGEMENTS}

I thank K.P.Deepesh for his generous assistance in the preparation of this manuscript and am grateful to the Indian Institute of Technology Madras for their financial support.

\section{REFERENCES}

1. S.G.Kamath, "An exact calculation of the Casimir energy in two planar models" in Frontiers of Physics - 2009, edited by Swee - Ping Chia, AIP Conference Proceedings 1150, American Institute of Physics,Melville,NY,2009,pp.402-406.

2. G.Bimonte et al. arXiv:hep-th/0310049v2.

3. G. Bimonte, E. Calloni, G. Esposito and L. Rosa, Phys.Rev.D 74, 085011(2006).

4. K.Bormann and F.Antonsen in Proceedings of the $3^{\text {rd }}$ Alexander Friedmann International Seminar,St.Petersburg 1995;arXiv:hep-th/9608142v1.

5. F.Antonsen and K.Bormann, "Propagators in Curved Space " arXiv:hep-th/9608141v1.

6. V.Fock, Phys. Z. Sowjetunion 12,404(1937).

7. J.Schwinger, Phy.Rev.82, 664(1951).

8. B.S.DeWitt, Phys.Rep.C19, 295(1975).

9. T.S.Bunch and L.Parker, Phys.Rev.D20, 2499(1979).

10. See Ref.5 especially the remarks following eq.(14) there.

11. S.Deser, R.Jackiw and G.'tHooft, Ann.Phys.120, 220(1984)

12. G.Clement, Int.J.Theor.Phys.24, 267(1985).

13. For example:

$e_{a}^{\mu}: e_{0}^{0}=\sin \alpha, e_{1}^{0}=i \cos \alpha, e_{2}^{0}=\frac{\lambda}{r} ; \quad e_{0}^{1}=i \frac{x}{r} \cos \alpha, e_{1}^{1}=\frac{x}{r} \sin \alpha, e_{2}^{1}=\frac{y}{r} ; e_{0}^{2}=i \frac{y}{r} \cos \alpha$, $\mathrm{e}_{1}^{2}=\frac{\mathrm{y}}{\mathrm{r}} \sin \alpha, e_{2}^{2}=-\frac{x}{r}$

$e_{\mu}^{a}: e_{0}^{0}=0, e_{0}^{1}=i, e_{0}^{2}=0 ; e_{1}^{0}=i \sin \alpha, e_{1}^{1}=-\frac{i \lambda y}{r^{2}}, e_{1}^{2}=\cos \alpha ; e_{2}^{0}=-i \cos \alpha, e_{2}^{1}=\frac{i \lambda x}{r^{2}}$,

$e_{2}^{2}=\sin \alpha ; \quad \alpha$ being a dimensionless parameter.

14. See for example, G. Barton, Elements of Green's functions and propagation (Oxford, Clarendon, 1989). 